TRANSACTIONS OF THE

AMERICAN MATHEMATICAL SOCIETY

Volume 185. November 1973

\title{
SQUARE INTEGRABLE REPRESENTATIONS OF NILPOTENT GROUPS
}

\author{
BY
}

\author{
CALVIN C. MOORE(1) AND JOSEPH A. WOLF(2)
}

\begin{abstract}
We study square integrable irreducible unitary representations (i.e. matrix coefficients are to be square integrable mod the center) of simply connected nilpotent Lie groups $N$, and determine which such groups have such representations. We show that if $N$ has one such square integrable representation, then almost all (with respect to Plancherel measure) irreducible representations are square integrable. We present a simple direct formula for the formal degrees of such representations, and give also an explicit simple version of the Plancherel formula. Finally if $\Gamma$ is a discrete uniform subgroup of $N$ we determine explicitly which square integrable representations of $N$ occur in $L_{2}(N / \Gamma)$, and we calculate the multiplicities which turn out to be formal degrees, suitably normalized.
\end{abstract}

1. Let $G$ be a locally compact unimodular group with center $Z$; we shall say that an irreducible unitary representation $\pi$ of $G$ on a Hilbert space $H(\pi)$ is square integrable if there are nonzero vectors $x_{1}$ and $x_{2}$ in $H(\pi)$ such that

$$
\int_{G / Z}\left|\left(\pi(s) x_{1}, x_{2}\right)\right|^{2} d \dot{\mu}(\dot{s})<\infty
$$

This formula requires some notes of explanation; if $z \in Z$, then by the irreducibility of $\pi, \pi(z)=\lambda(\pi)(z) \cdot 1$ where $\lambda(\pi)$ is a continuous homomorphism of $Z$ into the circle group $T$. It follows then that $\left(\pi(s z) x_{1}, x_{2}\right)=\lambda(\pi)(z)\left(\pi(s) x_{1}, x_{2}\right)$ and hence that the integrand in (*), as a function on $G$, is invariant under translation by elements of $Z$ and hence is really a function on $G / Z$. Finally $d \dot{\mu}(\dot{s})$ denotes integration over the group $G / Z$ with respect to a choice of Haar measure $\dot{\mu}$ on $G / Z$. The more standard notion of square integrability is that the absolute value squared of a matrix coefficient as in (*) should be integrable over the group $G$ itself, and if this happens we shall say that $\pi$ is square integrable in the strict sense. If $Z$ is compact, this is clearly the same as the present definition, but it is clear from our discussion that if $Z$ is not compact, the integral (*) taken over $G$ could not possibly converge as the integrand is constant on the cosets of a noncompact subgroup. There are many interesting examples such as reductive Lie groups and nilpotent Lie groups (the subject of this paper) where this extended definition is essential.

The usual equivalent characterizations of square integrability, together with the orthogonality relations, and the notion of formal degree carry over without

Received by the editors November 20, 1972.

AMS (MOS) subject classifications (1970). Primary 22D10, 22D30, 22E25, $22 \mathrm{E} 40$.

(1) Partially supported by NSF grant GP-30798.

(2) Partially supported by NSF grant GP-16651. 
essential change and we shall summarize these below. Our interest here is in simply connected nilpotent Lie groups and the study of their square integrable representations. In addition to abelian groups, the Heisenberg nilpotent groups have square integrable representations [16], and we shall characterize those nilpotent groups which have square integrable representations, and in those cases we shall also be able to give a very concrete description of them. Moreover we find an explicit formula for the formal degree of these representations (which incidentally is a direct generalization of Weyl's classical degree formula for the irreducible representations of a compact group, if both are viewed properly). It further turns out that, if there is one square integrable irreducible representation, then almost all irreducible representations (with respect to Plancherel measure) are square integrable and we shall display the Plancherel measure quite explicitly. In the final section we shall investigate nilpotent groups $N$ which have square integrable representations, and which have a discrete uniform subgroup $\Gamma$. We let $U$ be the usual representation on $L_{2}(N / \Gamma)$ and we exhibit a direct necessary and sufficient condition for a square integrable representation to be a summand. We also show that its multiplicity in $U$ is given, as one might hope, by its formal degree. Theorem 1 is without doubt "known" to many experts, but we include it first for completeness, and more importantly because some details of the proof will be essential later on. The other results through Theorem 6 are to some degree known; in particular Theorem 6 would follow from the pretty result announced in [18]; however no proof of this has been published. This paper of Kirillov came to our attention only after this paper had been written.

One by-product of our explicit formulas is that we are able to construct an example of a group with compact center having square integrable representations in the strict sense and for which there is no positive lower bound for the formal degrees-showing that in spite of some common examples, it is not always the case that the formal degrees are bounded from zero.

2. As in $\$ 1$, let $G$ be a unimodular locally compact group with center $Z$, and let $\pi$ be an irreducible representation. We associate to $\pi$ a character $\lambda(\pi)$ of the center $Z$ by $\pi(z)=\lambda(\pi)(z) \cdot 1$. We denote by $m(g, \phi, \psi)$ the matrix coefficient $(\pi(g) \phi, \psi)$ for $\phi, \psi \in H(\pi)$. We note that if $\lambda\left(\pi_{1}\right)=\lambda\left(\pi_{2}\right)$ and $\phi_{i}, \psi_{i} \in H\left(\pi_{i}\right)$, then $\left(\pi(g) \phi_{1}, \psi_{1}\right) \overline{\left(\pi(g) \phi_{2}, \psi_{2}\right)}$ is invariant under translation by $Z$ and is therefore a function on $G / Z$. We fix once and for all Haar measures $\mu$ on $G, \mu_{Z}$ on $Z, \dot{\mu}$ on $G / Z$ so that $d \mu=d \mu_{Z} d \dot{\mu}$. Finally for $\lambda \in \mathcal{Z}$ we denote by $U^{\lambda}$ the representation of $G$ induced by the representation $\lambda$ of $Z$. Recall that the Hilbert space of this representation consists of complex functions $h$ on $G$ satisfying $h(z g)=\lambda(z) h(g)$, square integrable on $G / Z$, and that $G$ operates by the formula $\left(U^{\lambda}(s) h\right)(g)$ $=h(g s)$. We now have the following well-known facts.

Theorem A. For an irreducible representation $\pi$ the following are equivalent.

(1) $\exists \phi_{1}, \phi_{2} \neq 0,\left|m\left(\cdot, \phi_{1}, \phi_{2}\right)\right| \in L_{2}(G / Z)$.

(2) $\left|m\left(\cdot, \phi_{1}, \phi_{2}\right)\right| \in L_{2}(G / Z)$ for all $\phi_{1}, \phi_{2} \in H(\pi)$.

(3) $\pi$ is a discrete summand of $U^{\lambda(\pi)}$. 
Theorem B. If the conditions of Theorem A are satisfied for an irreducible representation $\pi$, there is a positive number $d(\pi)$ such that

$$
\int_{G / Z} m\left(g, \phi_{1}, \psi_{1}\right) \overline{m\left(g, \phi_{2}, \psi_{2}\right)} d \dot{\mu}(\dot{g})=d(\pi)^{-1}\left(\phi_{1}, \phi_{2}\right) \overline{\left(\psi_{1}, \psi_{2}\right)} .
$$

If $\pi_{1}$ and $\pi_{2}$ are inequivalent irreducible representations satisfying the conditions of Theorem A, such that $\lambda\left(\pi_{1}\right)=\lambda\left(\pi_{2}\right)$, then

$$
\int_{G / Z}\left(\pi_{1}(g) \phi_{1}, \psi_{1}\right) \overline{\left(\pi_{2}(g) \phi_{2}, \psi_{2}\right)} d \dot{\mu}(g)=0 .
$$

The proofs are essentially well known and can be obtained as routine modifications of the theorems for square integrable representations in the strict sense. Even better we could appeal to Rieffel's general theory in [15] and obtain both as special cases.

The number $d(\pi)$ of Theorem B is called the formal degree of $\pi$. It of course depends on the choice of a Haar measure $\dot{\mu}$ in $G / Z$, and if $\dot{\mu}$ is replaced by $c \dot{\mu}$, the formal degrees of all representations change by a factor of $c^{-1}$. We now specialize to the case when $G=N$ is a connected and simply connected nilpotent Lie group with Lie algebra $n$. Let $z$ be the center of Lie algebra $n$, let $n^{*}$ be its dual vector space, and let $z^{\perp}$ be the annihilator of $z$ in $n^{*}$. According to the Kirillov theory ([6], [12]) the irreducible representations are parameterized by the space of $N$-orbits in $\mathfrak{n}^{*}$ under the coadjoint representation. We shall write $\pi(O)$ or $\pi(f)$ for the representation corresponding to an orbit $O$ or an $f \in O$. The character $\lambda(\pi(f))$ of $Z$ associated with this representation is $\lambda(\pi(f))(z)$ $=\exp (2 \pi i f(\log (z)))$. If $h$ is any other element of $\mathfrak{n}^{*}$ in the same orbit as $f$, then $f=h$ on $z$ and hence $O$ is contained in the affine hyperplane $f+z^{\perp}=h+z^{\perp}$. This hyperplane depends only on the restriction $y$ of $f$ to $z$, and we denote it by $H(y)$ for any $y \in z^{*}$ (the linear dual of $z$ ). The following provides the key to determine when representations are square integrable.

Proposition 1. Let $f \in \mathfrak{n}^{*}$, and let $O$ be its orbit under $N$, and let $\pi=\pi(f)$ $=\pi(O)$ be the corresponding representation. Let $\lambda(\pi)$ be the associated character of $Z$ and let $y$ be the restriction of $f$ to $z$. Then $\pi$ is a discrete summand of $U^{\lambda(\pi)}$ if and only if the orbit $O$ is equal to the hyperplane $H(y)$. In this case, $U^{\lambda(x)}$ is a primary representation.

Proof. As usual, we proceed by induction on the dimension of $N$, the result being obvious for abelian groups. If the dimension of $z$ is larger than one, there is a nonzero central subalgebra $z^{\circ}$ contained in the kernel of $f$. Its corresponding subgroup $Z^{\circ}$ is contained in the kernel of $\pi$. Now $\pi$ becomes a representation of $\pi^{\circ}$ of $N / Z^{\circ}$ whose corresponding linear functional $f^{\circ}$ is precisely $f$ when we regard $\left(\mathfrak{n} / z^{\circ}\right)^{*}$ as a linear subspace of $n^{*}$. Moreover the orbit $O^{\circ}$ of $f^{\circ}$ is precisely $O$ again viewing $\left(\mathfrak{n} / z^{\circ}\right)^{*}$ as a subspace of $n^{*}$. Suppose that $\pi$ is a direct summand of $U^{\lambda(x)}$; then as both of these representations vanish on $Z^{\circ}$, the corresponding 
representation $\pi^{\circ}$ of $N / Z^{\circ}$ is a summand of $U^{\lambda(x)}$ viewed as a representation of $N / Z^{\circ}$, and as such it is the representation of $N / Z^{\circ}$ induced by the representation $\lambda^{\circ}$ of $Z / Z^{\circ}$, where $\lambda^{\circ}\left(z Z^{\circ}\right)=\lambda(z)$, we shall write this as

$$
\sigma=\operatorname{ind}\left(Z / Z^{0}, N / Z^{0}, \lambda^{0}\right)
$$

denoting the induced representation. Since this induced representation $\sigma$ has an irreducible summand, it follows that $Z / Z^{\circ}$ is the entire center of $N / Z^{\circ}$ for otherwise $\sigma$ would admit a continuous direct integral decomposition over a space indexed by the dual of $A /\left(Z / Z^{\circ}\right)$ where $A$ is the entire center of $N / Z^{\circ}$. Thus $\sigma$ is the representation $U^{\lambda^{\circ}}$ of $N / Z^{\circ}$ determined by the character $\lambda^{\circ}$ of its center, and $\pi^{\circ}$ is a discrete summand of it. Since the proposition is true by induction for $N / Z^{\circ}$, we conclude that $O^{\circ}$ is the hyperplane $H\left(y^{\circ}\right)$ where $y^{\circ}=y$ when we view $\left(z / z^{\circ}\right)^{*}$ as a subspace of $z^{*}$. Since $O=O^{\circ}$ and $H\left(y^{\circ}\right)=H(y)$ with these identifications, we conclude that $O=H(y)$. Moreover since $U^{\lambda^{\bullet}}$ is primary, so is $U^{\lambda}$.

Conversely suppose that $O=H(y)$. Since $O=O^{\circ}$ it follows that the center of $n / z^{\circ}$ must be exactly $z / z^{\circ}$, otherwise $O^{\circ}$, an orbit, would be too big. It follows then that $H(y)=H\left(y^{\circ}\right)$ and hence $O^{\circ}=H\left(y^{\circ}\right)$, and then by induction $\pi^{\circ}$ is a discrete summand of $U^{\lambda^{\circ}}$. Finally we conclude that $\pi$ itself is a discrete summand of $U^{\lambda}$, completing this part of the argument.

We are now reduced to the case when the dimension of $z$ is one and $f(z) \neq 0$. Let $x$ be an element not in $z$ such that $[n, x] \subset z$ and let $n_{0}=\{u:[u, x]=0\}$. Then $n_{0}$ is an ideal of codimension one in $n$. We may arrange that $f(x)=0$ and we assume this done. Now we denote by $f_{0}$ the restriction of $f$ to $n_{0}$, and we let $O_{0}$ be its orbit in $\left(n_{0}\right)^{*}$. If $\pi_{0}$ is the corresponding representation of $N_{0}=\exp \left(n_{0}\right)$, it is part of the Kirillov theory that $\pi=\operatorname{ind}\left(N_{0}, N, \pi_{0}\right)$. Choose $y \notin n_{0}$ so that if $z=[x, y]$, then $f(z)=1$. Then the one parameter subgroup $(\exp (s y))$ is complementary to $N_{0}$. Moreover $\left(\operatorname{ad}^{*}(\exp (s y)) f\right)(x)=s$, and we let $f_{s}$ be the restriction of $\operatorname{ad}^{*}(\exp (s y)) f$ to $n_{0}^{*}$. Finally let $p$ be the projection of $n^{*}$ onto $n_{0}^{*}$ with kernel $n_{0}^{1}$.

Lemma 1. $p^{-1}\left(O_{s}\right)=\{f: f \in O, f(x)=s\}$ and $p\{f: f \in O, f(x)=s\}=O_{s}$.

Proof. This is in Kirillov [6].

Now suppose that $O=f+z^{\perp}=H(y)$ is an affine hyperplane of dimension $l-1$ where $l$ is the dimension of $n$. Then by the lemma, $O_{s}$ is an affine hyperplane for any value of $s$. More since $\operatorname{ad}^{*}(\exp (r X)) f-f$ vanishes on $n_{0}$, the mapping $p$ restricted to $O$ reduces dimensions by one. Therefore the dimension of the hyperplane $O_{s}$ is $(l-1)-2=l-3=l_{0}-2$ where $l_{0}$ is the dimension of $n_{0}$. Since $x$ and $z$ are both in the center of $n_{0}$ and since the center cannot have smaller index than the dimension of any orbit, it follows that the center $z_{0}$ of $n_{0}$ is precisely of dimension two, and so $z_{0}=(z)+(x)$. Thus $O_{s}=z_{0}^{\frac{1}{0}}+f_{s}$ for all values of $s$. 
Conversely suppose that $O_{s}=z_{0}^{\frac{1}{}}+f_{s}$ where $z_{0}$ is the span of $x$ and $z$. Then $O_{s}$ has dimension $l_{0}-2$, and hence by the lemma, $O$ has dimension $l-1$. Since $O$ is contained in an $l-1$ dimensional hyperplane $H(y)$ it is open in $H(y)$, but it is also closed in $H(y)[12]$ and hence equal to $H(y)$.

Let us summarize:

Lemma 2. $O=H(y)$ if and only if $O_{s}=3_{0}^{1}+f_{s}$ for one, or equivalently all, $s$. In this case $z_{0}$ is the center of $n_{0}$.

Now if $O=H(y), O_{s}=z_{0} \sigma^{\perp}+f_{s}$ by the lemma, and so by induction we know that $\pi_{s}$, the representation corresponding to $f_{s}$, is contained as a summand in $\sigma_{s}=\operatorname{ind}\left(Z_{0}, N_{0}, \lambda_{s}\right)$ where $\lambda_{s}(w)=\exp \left(2 \pi i f_{s}(\log w)\right)$ for $w \in Z_{0}$, and, moreover, $\sigma_{s}$ is primary for each $s$. Let us consider $p=\operatorname{ind}(Z, N, \lambda)$ which by induction in stages is $\operatorname{ind}\left(N_{0}, N\right.$, ind $\left.\left(Z_{0}, N_{0}, \operatorname{ind}\left(Z, Z_{0}, \lambda\right)\right)\right)$; the innermost representation, however, is rather clearly the integral

$$
\int \lambda_{s} d s
$$

where $d s$ is Lebesgue measure. Therefore the intermediate representation of $N_{0}$ above is the direct integral over the parameter $s$ of ind $\left(Z_{0}, N_{0}, \lambda_{s}\right)$ which by inductive assumption is primary and a multiple of $\pi_{s}$. Therefore the representation $U^{\lambda}$ is a direct integral over $s$ of multiples of ind $\left(N_{0}, N, \pi_{s}\right)$. However, by Kirillov theory, $\operatorname{ind}\left(N_{0}, N, \pi_{s}\right)$ is $\pi$ for all $s$, and so $U^{\lambda}$ is a multiple of $\pi$ and is primary, and in particular $\pi$ occurs as a summand in $U^{\lambda}$. This proves the proposition in one direction.

Suppose now that $\pi$ is a summand of the representation $U^{\lambda}=\operatorname{ind}(Z, N, \lambda)$. The restriction of $\pi$ to $N_{0}$ is by Kirillov theory the direct integral over the parameter $s$ of the representations $\pi_{s}$ described above. On the other hand, the restriction of $U^{\lambda}$ to $N_{0}$ is, by Mackey's subgroup theorem [8], an infinite multiple of ind $\left(Z, N_{0}, \lambda\right)$. This latter representation is by induction in stages the same as ind $\left(Z_{0}, N_{0}\right.$, ind $\left.\left(Z, Z_{0}, \lambda\right)\right)$, and thus is the direct integral over the parameter $s$ of the representations $\rho_{s}=\operatorname{ind}\left(Z_{0}, N_{0}, \lambda_{s}\right)$. To summarize, the direct integral over $s$ of representations $\pi_{s}$ occurs as a summand in the integral of the representations $\rho_{s}$ and we note that on $Z_{0}$, which is central in $N_{0}, \rho_{s}$ and $\pi_{s}$ are both multiples of the character $\lambda_{s}$. It follows now from direct integral theory, using crucially the fact that $Z$ is central, that $\pi_{s}$ is a summand of $\rho_{s}$ for almost all $s$ (Lebesgue measure in $s$ ).

Now since there is a one parameter group $\phi(t)=\operatorname{ad}(\exp (t y))$ of automorphisms such that $\phi(t) \cdot \pi_{s}=\pi_{s+t}$ and $\phi(t) \cdot \rho_{s}=\rho_{t+s}$ it follows that if $\pi_{s}$ is a summand of $\rho_{s}$ for one value of $s$, the same is true for all $s$. Let $C$ be the center of $N_{0}$; if $C \neq Z_{0}$ then ind $\left(Z_{0}, N_{0}, \lambda_{s}\right)=\operatorname{ind}\left(C, N_{0}\right.$,ind $\left.\left(Z_{0}, C, \lambda_{s}\right)\right)$ can be displayed as a continuous integral over the dual group of the vector space $C / Z_{0}$. Thus $Z_{0}$ is the center of $N_{0}$ and $\pi_{0}$ is a summand of ind $\left(Z_{0}, Z, \lambda_{0}\right)$. By induction it follows that the orbit $O_{0}$ of $f_{0}$ is a hyperplane $H\left(y_{0}\right)$ of codimension two. It 
follows now from Lemma 1 that $O$ is equal to the hyperplane $H(y)$ of codimension one in $\mathfrak{n}^{*}$, and this completes the proof of the proposition.

Suppose that $f$ is any element of $\mathfrak{n}^{*}$; we define a skew symmetric bilinear form $b_{f}$ on $\mathfrak{n}$ by $b_{f}(x, y)=f([x, y])$. The singular subspace of $b_{f}$ clearly contains $z$ and so $b_{f}$ may be viewed as a skew two-form on the quotient space $n / z$. In terms of this we summarize our results in the following statement.

Theorem 1. For a linear functional $f$ on $\mathfrak{n}$, with orbit $O$ and corresponding representation $\pi$, the following are equivalent.

(1) $\pi$ is square integrable.

(2) ind $(Z, N, \lambda)$ is primary where $\lambda(z)=\exp (2 \pi i f(\log z)), z \in Z$, the center of N.

(3) $O=f+z^{\perp}=H(y)$ where $y$ is the restriction of $f$ to $z$.

(4) $b_{f}$ is nondegenerate on $\mathfrak{n} / \mathfrak{z}$.

Proof. The statement $1 \Leftrightarrow 2$ follows from Theorem $A$ and Proposition 1; $2 \Leftrightarrow 3$ also follows from Proposition 1. For the rest, it is quickest to prove $3 \Leftrightarrow 4$. Therefore let $\mathfrak{h}$ be the singular subspace of $b_{f}$ so $\mathfrak{h}=\{x \mid f[x, y]=0 \forall y \in \mathfrak{n}\}$ is well known to be the Lie algebra of the stability subgroup at $f$ in the coadjoint representation. It follows that the dimension of $O$ is precisely the codimension of $\mathfrak{h}$. Thus if $O=H(y), O$ has dimension equal to the codimension of $z$ and so $\operatorname{dim}(\mathfrak{h})=\operatorname{dim}(\mathfrak{z})$ and since $\mathfrak{h} \supset \mathfrak{z}$, we have $\mathfrak{h}=\mathfrak{z}$ and $b_{f}$ is nondegenerate on $\mathfrak{n} / \mathfrak{z}$. If $b_{f}$ is nondegenerate there, we see that $\operatorname{dim}(O)=\operatorname{dim}(H(y))$ and hence by invariance of domain and the fact that $O$ is closed, it is equal to $H(y)$ and we are done.

3. We now proceed to describe in more detail the parameterization of all square integrable representations of a group $N$. We need some preliminary material about bilinear forms for this first, so let $V$ be a vector space over $R$ of dimension $n$ with a fixed volume element $\alpha$ (an alternating $n$-linear form). Suppose that $b$ is a skew symmetric bilinear form on $V$; we recall the definition of the Pfaffian $\operatorname{Pf}(b)$ of $b$. If $n$ is odd, we let $\operatorname{Pf}(b)=0$, and if $n=2 m$ is even, the $m$ th exterior power $b^{m}$ is a multiple of $\alpha$, and we define $\operatorname{Pf}(b)$ by $b^{m}=\operatorname{Pf}(b) \alpha$. This of course depends on $\alpha$ but only up to a fixed scalar independent of $b$. We may also define the determinant of $b$ relative to $\alpha$ and note that $\operatorname{det}(b)=(\operatorname{Pf}(b))^{2}$ so that $\mathrm{Pf}$ is a square root of the determinant on skew symmetric forms. We note that $\mathrm{Pf}$ is a homogeneous polynomial of degree $m$ on the space of skew symmetric forms. We note that for the most part we shall only be interested in the absolute value of the Pfaffian, and this is determined unambiguously by the measure on $V$ associated to the alternating $n$ form $\alpha$ rather than by $\alpha$ itself.

The following construction will be useful presently: let $\alpha$ and $V$ be as above and let $V^{*}$ be the dual vector space, and let $\alpha^{*}$ be a volume form so that the Fourier transform $f \rightarrow \hat{f}$ from $L^{2}(V)$ into $L^{2}\left(V^{*}\right)$ is an isometry where

$$
\hat{f}(x)=\int f(v) \exp (2 \pi i x(v)) d \alpha(v) \text { for } x \in V^{*} .
$$


(Note that $\alpha^{*}$ is determined up to a sign.) Let $b$ be a nondegenerate skew twoform on $V$ and let $t(b)$ be the linear isomorphism of $V$ into $V^{*}$ determined by $(t(b) v)(w)=b(v, w)$. We transport $b$ to $V^{*}$ by the formula $b^{*}(x, y)=$ $b\left(t(b)^{-1} x, t(b)^{-1} y\right)$ so that $b^{*}$ is a skew two-form on $V^{*}$ and we want to compare the Pfaffian of $b$ (relative to $\alpha$ ) with the Pfaffian of $b^{*}$ relative to $\alpha^{*}$ or rather their absolute values.

Lemma 3.1. We have $\left|\operatorname{Pf}(b) \cdot \operatorname{Pf}\left(b^{*}\right)\right|=1$.

Proof. This is a routine calculation which we omit, and note only that if $\left\{e_{i}\right\}$ is a basis for $V$ and $\alpha\left(e_{i}, \ldots, e_{n}\right)= \pm 1$, and if $\left\{x_{i}\right\}$ is the dual basis, $\alpha^{*}\left(x_{i}, \ldots, x_{n}\right)$ $= \pm 1$.

We specialize these considerations to the case when $V=\mathfrak{n} / \mathfrak{z}$ where $\mathfrak{n}$ is a nilpotent Lie algebra, $z$ its center and where $b=b_{f}\left(b_{f}(x, y)=f([x, y])\right)$ viewed as a two-form on $\mathfrak{n} / \mathrm{z}$. We define $P(f)=\operatorname{Pf}\left(b_{f}\right)$ and note that this is a homogeneous polynomial function on $\mathfrak{n}^{*}$.

Lemma 3.2. The function $P(f)$ depends only on the restriction $y$ of $f$ to $z$, and hence there is a homogeneous polynomial function on $z^{*}$, also denoted by $P$, so that $P(f)=P(y)$.

Proof. Suppose that $P(f) \neq 0$ so that $b_{f}$ is nondegenerate on $n / z$. Then according to Theorem 1 , the $N$-orbit $O$ of $f$ is the entire hyperplane $f+z^{\perp}$ $=H(y)$ where $y$ is the restriction of $f$ to $z$. We have to show that if $g$ has the same restriction to $z$ as does $f$ then $P(f)=P(g)$. But we know that there exists $n \in N$ with $\operatorname{ad}^{*}(n) f=g$, and it follows that $b_{g}(x, y)=b_{f}\left(\operatorname{ad}(n)^{-1} x, \operatorname{ad}(n)^{-1} y\right)$ so that $b_{g}=n \cdot b_{f}$ in terms of the induced action of $N$ on two-forms. On the other hand, since the action of $N$ preserves any alternating $n$-linear form as $N$ is unimodular and connected, and since $\mathrm{Pf}$ is an invariant polynomial, it follows that $b_{f}$ and $b_{g}$ have the same Pfaffian and so $P(f)=P(g)$.

If however $P(f)=0$, and if $g$ is another linear functional whose restriction to $z$ is the same as that of $f$, we must also have $P(g)=0$. For if $P(g) \neq 0$, the above argument would show that $P(f)=P(g) \neq 0$, a contradiction. This completes the proof of the lemma.

This lemma of course provides another criterion for the representation $\pi(f)$ associated to a linear functional $f$ to be square integrable, namely that $P(f) \neq 0$. To each irreducible representation $\pi(f)$ we attach to $\pi$ the restriction $y$ of $f$ to $z$, or equivalently the character $\lambda, \lambda(z)=\exp (2 \pi i f(\log z))$ of $Z$. On the other hand if $y \in z^{*}$ and if $P(y) \neq 0$, there is a linear functional $f$ extending $y$ with $P(f) \neq 0$. If $\lambda(y)$ is the character of $Z$ corresponding to $y$ given by $\lambda(y)(z)$ $=\exp (2 \pi i y(\log z))$ then ind $(Z, N, \lambda(y))$ is, by Theorem 1 , a primary representation which is a multiple of the square integrable representation $\pi(f)$. We let $\phi(y)$ be this representation $\pi(f)$. Finally let $V=\{y: P(y)=0\}$ be the zero set of $P$. 
Theorem 2. The map $\phi$ is a bijection of the set $\left\{y \in z^{*}, P(y) \neq 0\right\}=z^{*}-V$ onto the set of square integrable irreducible representations of $N$. Moreover $\phi(y)$ is the only irreducible representation of $N$ whose associated character $\lambda(\pi)$ on $Z$ is $\lambda(y)$. Finally $\phi$ is a homeomorphism from the natural topology of $z^{*}-V$ to the Fell topology on representations.

Proof. By Theorem 1, $\phi$ maps $z^{*}-V$ onto the set of all square integrable representations. Now if $y \in z^{*}-V$ and if $\pi$ is an irreducible representation with $\pi(z)=\exp (2 \pi i y(\log z))$, the associated orbit of $\pi$ must lie in the hyperplane $H(y)$, but by Theorem $1, H(y)$ is an orbit, namely the orbit associated to $\phi(y)$. Thus $\pi=\phi(y)$, and this shows that $\phi$ is injective and establishes the second statement of the theorem.

To see that $\phi$ is continuous, note that $y \rightarrow \lambda(y)$ is a homeomorphism of $z^{*}-v$ into $\mathcal{Z}$, and that $\lambda \rightarrow \operatorname{ind}(Z, N, \lambda)$ is continuous from $Z$ into the Fell topology on representations of $N$ [3]. Finally since this induced representation for $\lambda=\lambda(y)$ is a multiple of $\phi(y)$, these have the same kernel in the associated $C^{*}$ algebra, and so the map ind $(Z, N, \lambda(y)) \rightarrow \phi(y)$ is continuous. Thus $\phi$ is continuous since it is the composition of continuous maps. On the other hand the map which associates the character $\lambda(\pi)$ on $Z$ to a representation $\pi$ is clearly continuous as it is defined simply by restricting a representation to a subgroup. Finally we have already noted that the inverse of $y \rightarrow \lambda(y)$ is continuous and it follows now that $\phi^{-1}$ is continuous, completing the proof.

Remark. The homogeneous polynomial $\boldsymbol{P}$ can be viewed in several different ways; we have already seen that it is equivalently a function on $n^{*}$ or on $z^{*}$. But also we may view it as an element of the symmetric algebra $S(z)$ on the center, as $S(z)$ can always be viewed as polynomial functions on $z^{*}$. Finally let $\mathfrak{U}$ be the universal enveloping algebra of $n$, and let 3 be its center. We note that the center $z$ of $n$ is naturally contained in 3 , and hence also $S(z)$ is naturally contained in 3. Thus $P$ may in addition be viewed as an element of center of the universal enveloping algebra of $n$. (Of course $P$ is only determined up to a scalar multiple.)

We shall now give a structural characterization of those $N$ which have square integrable representations. Theorem 1 of course does this but the following is of a slightly different nature; recall from the above remark that $S(z)$ is naturally included in 3 .

Theorem 3. The group $N$ has square integrable representations if and only if $S(z)=3$.

Proof. If $N$ has square integrable representations, $P$ is not the zero polynomial and, by Theorem $1, P(f) \neq 0$ implies that the $N$-orbit of $f$ is the hyperplane $f+z^{\perp}$. It is well known that by symmetrization the enveloping algebra $\mathfrak{U}$ can be realized as all the polynomials on $\mathfrak{n}^{*}$ so that 3 is realized precisely as the $N$ invariant ones. Under this isomorphism $S(z)$ is realized as those polynomials coming from polynomials on $z^{*}$ via the projection map of $n^{*}$ onto $z^{*}$ with kernel 
$z^{\perp}$. Equivalently, $S(z)$ may be viewed as those polynomials invariant under translation by elements of $z^{\perp}$. Therefore, we must show simply that any $N$ invariant polynomial on $n^{*}$ is invariant under translations by $z^{\perp}$. Let $Q$ be $N$. invariant and let $k \in z^{\perp}$ and consider the polynomial $R(x)=Q(x+k)-Q(x)$. Now if $P(x) \neq 0, x$ and $x+k$ are in the same $N$-orbit by Theorem 1 and so $R(x)=0$. Therefore $P R=0$, and since we have an integral domain and since $P \neq 0$ by hypothesis, $R=0$ and we are done.

Conversely suppose that $3=S(z)$, and let $\operatorname{dim} n=n$ and $\operatorname{dim} z=r$. If $K$ is the field of fractions of 3 , it evidently has transcendence degree $r$ as it is the field of rational functions in $r$ variables. According to [2], the dimension of the generic orbit of $N$ in $n^{*}$ is the dimension of $n$ minus the transcendence degree of $K$, or in other words $n-r$ in this case. This says that the generic isotropy group has dimension $r$, and hence, as we have argued before, it follows that this isotropy group is the center, and then by Theorem $1, N$ has square integrable representations.

We pause now to consider some examples of groups that satisfy the conditions we have been discussing.

Example 1. Let $\mathfrak{n}$ be the $2 n+1$ dimensional Heisenberg algebra with basis $x_{1}, \ldots, x_{n}, y_{1}, \ldots, y_{n}, z$ with $\left[x_{i}, y_{j}\right]=z$ and all other brackets zero. Then $z$ is one dimensional and spanned by $z$, and let $z^{*}$ be the element of $z^{*}$ dual to $z$. We pick the volume element $\alpha$ on $\mathfrak{n} / \mathrm{z}$ so that $\alpha\left(x_{1}, \ldots, y_{n}\right)=1$, and then it is easy to see that $P\left(t z^{*}\right)=t^{n}$. Thus every infinite dimensional representation is square integrable, a fact which is well known.

Example 2. Let $a_{i j}$ be any $n$ by $n$ matrix and construct a Lie algebra of dimension $2 n+2$ with basis $x_{1}, \ldots, x_{n}, y_{1}, \ldots, y_{n}, z, w$ such that $\left[x_{i}, y_{j}\right]$ $=a_{0} \delta_{i j} z-a_{i j} w, a_{0} \in R$, and all other brackets zero. The center is spanned by $z$ and $w$ and linear functionals may be described by $f(z)=u, f(w)=v$ so that functions on $z^{*}$ can be taken to be functions of $u$ and $v$. It is not hard to verify that $P(u, v)=\operatorname{det}\left(a_{0} u-v a_{i j}\right)$, or in other words the characteristic polynomial of the matrix $a_{i j}$ evaluated at $a_{0}(u / v)$ and multiplied by $v^{n}$. Evidently we may then arrange by suitable choice of $a_{i j}$ that $P$ be an arbitrary homogeneous polynomial in two variables.

Let us also add the remark that one can show that there is no upper bound on the nilpotent length of a nilpotent group which has square integrable representations.

4. In Theorem 2 we gave a parameterization of the square integrable representations of $N$, when there were any, by the map $\phi$ from $z^{*}-V$ into $\hat{N}$. The polynomial $P$ played perhaps an auxiliary role in defining the exceptional set $\gamma$, but we shall see that it is of fundamental importance in its own right because it gives us the formal degree of the square integrable representations.

Theorem 4. The formal degree of the square integrable representation $\pi(h)=\phi(h)$ given by Theorem 2 is $d(\pi(h))=|P(h)|$ for $h \in z^{*}-\mathcal{V}$. 
Let us defer the proof for a moment. We note that $d(\pi)$ depends on a choice of Haar measure on $N / Z$, but also $|P|$ depends on a choice of a volume element on $\mathfrak{n} / \mathrm{z}$ which is the same thing as a measure on $N / Z$. It is understood in Theorem 4 that the measure used to compute $d(\pi)$ must come from the volume element in $\mathfrak{n} / \mathfrak{z}$ used to define $P$. The statement of the theorem is therefore intrinsic and independent of any such choice.

As we have remarked before, we may view $P$ as an element of the center 3 of the universal enveloping algebra of $\mathfrak{n}$. Then any irreducible representation $\pi$ has infinitesimal character $\chi_{\pi}$ which is a homomorphism of 3 into the complex numbers. On $z \subset S(z), \chi_{\pi}$ is given by $\chi_{\pi}(z)=h(z)$ if $\pi(\exp (z))=\exp (2 \pi i h(z))$. We now have the following formulation.

Theorem 5. The formal degree $d(\pi)$ of any irreducible representation is given by $\left|\chi_{\pi}(P)\right|$ where we understand the formal degree of a non square integrable representation to be zero.

Theorem 5 is true for any nilpotent group and is of course an immediate corollary of Theorem 4 . We might note that the theorem could be written as $d(\pi)^{2}=\left|\chi_{\pi}(P)\right|^{2}=\chi_{\pi}(P \bar{P})$ where $a \rightarrow \bar{a}$ is the conjugate linear antiautomorphism of the universal enveloping algebra extending the map $x \rightarrow-x$ of $\mathfrak{n}$. As such it is a direct extension of an observation of Harish-Chandra in [4, p. 40], that the squares of the degrees of the representations of a compact group could be read off in this way from an element of the center of the enveloping algebra.

Indeed the similarity with compact groups goes even deeper, for let us write Weyl's degree formula for a representation in terms of its highest weight $\Delta$ relative to a fixed Cartan subalgebra as

$$
d(\pi)=\prod_{\alpha>0}(\Lambda+\rho, \alpha) /(\rho, \alpha)
$$

where the product is taken over all positive roots and where $\rho$ is half the sum of the positive roots. Now according to general principles of Kostant, the representation $\pi$ of highest weight $\Lambda$ corresponds to a linear functional $f(\pi)$ on the Lie algebra in a fashion entirely analogous to the nilpotent case. Indeed $f(\pi)$ may be taken to be $\Lambda+\rho$ on the Cartan subgroup in question and zero on the orthogonal complement of it, and now a moment's thought shows that the righthand side of (*) is a formula for the Pfaffian (relative to a fixed volume element) of the skew two-form $b_{f(x)}$ which is defined in exactly the same way as above. The analogy is then complete and Theorems 4 and 5 may be viewed as direct generalizations of the Weyl degree formula for compact groups.

These considerations immediately suggest that such formulas for formal degrees should have a quite general validity at least to the extent of the validity of the Kostant principle which says that there should be some kind of correspondence between linear functionals on the Lie algebra of a group $G$ and irreducible representations of $G$. Indeed one may plausibly conjecture when a 
representation $\pi(f)$ associated with a linear functional is square integrable as follows: Let $G_{f}$ be the isotropy group of $f$ in $G$; then $\pi(f)$ should be square integrable or otherwise "discrete" if and only if $G_{f} / Z$ is compact where $Z$ is the center of $G$. This is precisely true for nilpotent and compact groups. It is also true for connected reductive groups if one associates linear functionals to the known representations of semisimple Lie groups in the usual way. The results in [13] suggest that it should be true for solvable groups. One would also conjecture that the formal degree of $\pi(f)$ would have something to do with the Pfaffian of $b_{f}$. For nonunimodular groups, one has to modify the definition of square integrability and the notion of formal degree, but this is reasonably well understood now ([7], [10], [13], [17]). The formal degree becomes a selfadjoint operator in this case, but that simply means that the analogue of the Pfaffian polynomial $P$ cannot be expected to lie in the center of the enveloping algebra in the nonunimodular case, but rather should be a semi-invariant.

After these extended remarks of a conjectural nature, let us return to the subject proper under discussion. Our next theorem describes in explicit terms the Plancherel measure on $\hat{N}$. For this we need a normalization of Haar measure $\mu_{N}$ on $N$ which together with our choice of measure on $N / Z$ gives a choice of Haar measure $\mu_{Z}$ on $Z$. These choices give rise to a choice of Lebesgue measure $d n$, on $\mathfrak{n}, d z$ on $\mathfrak{z}$ and $d \dot{n}$ on $\mathfrak{n} / \mathfrak{z}$ so that $d n=d \dot{n} d z$ symbolically. In turn we shall demand that the Fourier transform of functions on $n, z$ or $n / z$ into functions on the linear duals $\mathfrak{n}^{*}, \mathfrak{z}^{*},(\mathfrak{n} / \mathfrak{z})^{*}$ be an isometry, and this determines a normalization of Lebesgue meausre $d n^{*}, d z^{*}$ and $d \nu$ on $n^{*}, z^{*}$, and $(\mathrm{n} / \mathrm{z})^{*}$ respectively. Symbolically we have $d n^{*}=d z^{*} d \nu$.

The following is just what one might expect.

Theorem 6. If $N$ has square integrable irreducible representations, Plancherel measure is concentrated on the set of square integrable representations and when transported via the map $\phi$ of Theorem 2 to $z^{*}-\gamma$, Plancherel measure is $c|P(x)| d z^{*}(x)$ where $P$ is as before, and $c=n ! 2^{n}$, where $2 n$ is the generic dimension of the orbits in $\mathfrak{n}^{*}$.

The rest of this section will be devoted to the proofs of Theorems 4 and 6. For the proof of Theorem 4 we proceed by induction on the dimension of $n$. First of all, we fix a square integrable representation $\pi(h)$ of $N$, and if the dimension of the center $z$ of $\mathfrak{n}$ is larger than one there is a nonzero central subalgebra $z^{\circ}$ with corresponding subgroup $Z^{\circ}$ such that $h=0$ on $z^{\circ}$ and $\pi(h)\left(Z^{\circ}\right)=1$. We replace $h$ and $\pi(h)$ by the corresponding $h^{\circ} \in\left(z / z^{\circ}\right)^{*} \subset z^{*}$ and $\pi\left(h^{\circ}\right)$ of $N / Z^{\circ}$. We note as in Theorem 1 that $z / z^{\circ}$ is the center of $n / z$, and we apply our inductive assumption to $h^{\circ}, \pi\left(h^{\circ}\right)$ and $n / z^{\circ}$. The desired assertion for $\pi(h)$ now follows immediately as $d\left(\pi\left(h^{\circ}\right)\right)=d(\pi(h))$ and $P\left(h^{\circ}\right)=P(h)$.

We are reduced to the case when $z$ has dimension one and $h(z) \neq 0$. As in the proof of Theorem 1, we select an element $x \notin z$ with $[n, x] \subset z$ and let 
$\mathrm{n}_{0}=\{u \mid[u, x]=0\}$ and select $y \notin \mathrm{n}_{0}$ such that $[y, x]=z$ where $z \in z$ with $h(z)=1$. Let $f$ be any linear functional on $n$ restricting to $h$ on $z$ so that $f(x)=0$, and let $f_{0}$ be its restriction to $n_{0}$ and $\pi_{0}$ the corresponding representation. As in Theorem 1, conjugation of $f_{0}$ and $\pi_{0}$ by the one parameter group $\exp (s y)$ gives rise to a one parameter family $\pi_{s}$ of representations of $N_{0}$ with linear functionals $f_{s}$ where $f_{s}(x)=s$. The proof of Theorem 1 also provides the very important piece of information that $z_{0}=z+(x)$ is the center of $n_{0}$. Now fix a volume element $\alpha_{0}$ on $n_{0} / z_{0}$ and construct the Pfaffian polynomial $P_{0}$. We also choose a volume element $\alpha$ on $n / z$ as follows: Choose a linear subspace $V$ of $n_{0}$ complementary to $(x)$ and containing $z$. Then $V / z$ is naturally isomorphic via a projection map $j$ to $\mathfrak{n}_{0} / z_{0}$. Then $\mathfrak{n} / \mathfrak{z} \approx V / z \oplus(x) \oplus(y)$ and $V / z$ and $x$ are orthogonal with respect to the form $b_{f}$. By replacing $y$ by $y+u$ for some $u$ in $V$ we may ensure that $y$ is orthogonal to $V$ with respect to $b_{f}$ and we assume this done as it does not change anything done previously. Then $V / z$ is orthogonal to $(x)+(y)$ with respect to $b_{f}$ and we define the $n$-linear form on $n / z \approx V / z \oplus(x) \oplus(y)$ by $\alpha\left(j^{-1}\left(u_{1}\right) \cdots j^{-1}\left(u_{k}\right), x, y\right)=\alpha_{0}\left(u_{1}, \ldots u_{k}\right)$ where the $u_{i}$ are in $n_{0} / z_{0}$. Now since $b_{f}(x, y)=1$, and $b_{f}\left(j^{-1}\left(u_{i}\right), j^{-1}\left(u_{j}\right)\right)=b_{f_{0}}\left(u_{i}, u_{j}\right)$, it follows that $P(f)=P_{0}\left(f_{0}\right)$. Moreover, since $f_{s}$ is conjugate to $f$ by the volume preserving transformation $\operatorname{ad}^{*}(\exp (s y)), P_{0}\left(f_{0}\right)=P_{0}\left(f_{s}\right)$ for all $s$.

Now by our inductive assumption the formal degrees $d\left(\pi_{s}\right)$ are all equal to $P_{0}\left(f_{0}\right)=P(f)$. We write $N$ as the semidirect product of $N_{0}$ and the one parameter subgroup $\{\exp (s y)\}$ and then we may realize $\pi=\operatorname{ind}\left(N_{0}, N, \pi_{0}\right)$ on the Hilbert space $L_{2}\left(R, H\left(\pi_{0}\right)\right)$ of square integrable functions on the line with values in the Hilbert space of $\pi_{0}$. (This is achieved by restricting functions in the induced representation space to the line given by the one parameter group $\{\exp (s y)\}$.) The representation $\pi$ then has the form

$$
\left(\pi\left(n_{0} \exp (t y)\right) \phi\right)(s)=\pi_{0}\left(s \cdot n_{0}\right) \phi(s+t)=\pi_{s}\left(n_{0}\right) \phi(s+t)
$$

where $s: n_{0}=\exp (s y) n_{0} \exp (-s y)$. We fix a $u \in H\left(\pi_{0}\right)$ and consider a function $\phi$ in $L_{2}\left(R, H\left(\pi_{0}\right)\right)$ of the form $\phi(s)=q(s) u$ where $q$ is a scalar function. Now the matrix coefficient $\left(\pi\left(n_{0} \exp (t y)\right) \phi, \phi\right)$ is given by

$$
\int_{-\infty}^{\infty}\left(\pi_{0}\left(s \cdot n_{0}\right) u, u\right) q(s+t) \overline{q(s)} d s
$$

This function is to be integrated over $N / Z$ and we do so by pulling it back to $\mathfrak{n} / \mathrm{z}$. More precisely $\mathfrak{n} / \mathrm{z} \approx V / \mathfrak{z} \oplus(x) \oplus(y)$ and define a map from $n / z$ to $N / Z$ by $(\dot{v}+r X+t y) \mapsto \exp (\dot{v}) \exp (r x) \exp (t y)$ where $\dot{v} \in V / z$ and $\exp (\dot{v})$ is the obvious element of $\exp (V) / Z \subset N / Z$. Under this map it is clear that Lebesgue measure goes over into Haar measure on $N / Z$ and that our normalizations are correct if we use $d \dot{v} d t d r$ on $\mathrm{n} / \mathrm{z}$ where $d \dot{v}$ is correctly normalized by the isomorphism of $V / z$ with $n_{0} / z_{0}$. We have to evaluate the following integral: 
(*) $\int_{N / Z}|(\pi(n) \phi, \phi)|^{2} d \dot{n}$

$$
=\int_{-\infty}^{\infty} \int_{-\infty}^{\infty} \int_{V / 8}\left|\int_{-\infty}^{\infty} \pi_{0}(s \cdot(\exp (\dot{v}) \exp (r X)) u, u) q(s+t) \overline{q(s)} d s\right|^{2} d \dot{v} d r d t
$$

The innermost integrand is for fixed $s$ a function $\psi(\dot{v}, r, t)$, and as

$$
\begin{aligned}
s \cdot(\exp (\dot{v}) \exp (r X)) & =(s \cdot \exp (\dot{v}))(s \cdot \exp (r X)) \\
& =(s \cdot \exp (\dot{v})) \exp (r X) \exp (r s z)
\end{aligned}
$$

and as

$$
\pi_{0}(\exp (r s z)) u=\exp (2 \pi i r s) u,
$$

$\psi(\dot{v}, r, t)=\psi(\dot{v}, 0, t) \exp (2 \pi i r s)$ is periodic. We interchange the $\dot{v}$ and $r$ integration and the inner part of $(*)$ is then

$$
\int\left|\int_{-\infty}^{\infty} \psi(\dot{v}, 0, t) q(s+t) \overline{q(s)} \exp (2 \pi i r s) d s\right|^{2} d r
$$

which may be evaluated by the Plancherel formula for the line and is equal to

$$
\int_{-\infty}^{\infty}|\psi(\dot{v}, 0, t) q(s+t) \overline{q(s)}|^{2} d s
$$

Thus (*) is

$$
\int_{-\infty}^{\infty} \int_{V / s} \int_{-\infty}^{\infty}\left|\left(\pi_{s}(\exp (\dot{v})) u, u\right) q(s+t) \overline{q(s)}\right|^{2} d s d \dot{v} d t
$$

We interchange order of integration and perform the $\dot{v}$ integration first and the result of that integration is recognizable as

$$
\int_{V / s}\left|\left(\pi_{s}(\exp (\dot{v})) u, u\right)\right|^{2} d \dot{v}=d\left(\pi_{s}\right)^{-1}(u, u)^{2}
$$

which is independent of $s$ as noted above and equal to $|P(f)|^{-1}(u, u)^{2}$ by induction. The entire integral is then

$$
|P(f)|^{-1}(u, u)^{2} \int_{-\infty}^{\infty} \int_{-\infty}^{\infty}|q(s+t) \overline{q(s)}|^{2} d s d t
$$

which is $|P(f)|^{-1}(u, u)^{2}(q, q)^{2}=|P(f)|^{-1}(\phi, \phi)^{2}$ as desired. This completes the proof.

We now turn to the proof of Theorem 6. First let us note that the Plancherel measure on $\hat{N}$ is concentrated on the set of square integrable representations, provided that that set is nonvoid. For if $h$ is a linear functional on $z$, the center of $n$, let $\lambda(h)$ be the corresponding character of $Z, \lambda(h)(\exp (z))=\exp (2 \pi i h(z))$, 
and let $U(h)$ be representation of $N$ induced by $\lambda(h)$. By the theorem on induction in stages the regular representation $\pi$ is the direct integral of the $U(h)$ with respect to Haar measure $d z^{*}(h)$ on $z^{*}$. Since the Pfaffian polynomial is nonzero, its zero set is of measure zero and so we can replace $z^{*}$ by $z^{*}-V=\{h: P(h) \neq 0\}$ and, for $h$ in this set, $U(h)$ is a multiple $\operatorname{dim} \pi(h)(\pi(h))$ of the irreducible square integrable representation $\pi(h)$ corresponding to $h$. Then

$$
\pi=\int_{\partial^{*}-\nu}(\operatorname{dim} \pi(h)) \pi(h) d z^{*}(h)
$$

is the central decomposition of $\pi$, showing that Plancherel measure is concentrated on the square integrable representations.

To compute the measure precisely, let $\phi_{1}$ be a $C^{\infty}$ function of compact support on $N$, and let $\phi_{1}(n)=\phi(\exp (n))$ be the corresponding function on $\mathfrak{n}$. Plancherel measure $\mu$ in this case may be viewed as a measure on $z^{*}-\nu$ such that

$$
\begin{aligned}
\phi(0) & =\phi_{1}(e)=\int_{z^{\bullet}-\nu} \operatorname{Trace}(\pi(h)(\phi)) d \mu(h) \\
& =\int_{3^{\bullet}-\nu} \Theta_{\pi(h)}(\phi) d \mu(h)
\end{aligned}
$$

where $\Theta_{\pi(h)}$ is the distribution character of $\pi(h)$. But according to [11], $\Theta_{\pi(h)}$ is obtained as follows in this case. Let $O(h)=H(h)$ be the corresponding orbit (which is an affine hyperplane). The group action of $N$ on $O(h)$ gives rise to a linear isomorphism $j(h)$ of $n / z$ ( $z$ being the isotropy algebra of $f$ ) onto the tangent space of the orbit $O(h)=H(y)$. The tangent space of the hyperplane $H(y)$ is of course precisely $z^{\perp} \subset \mathfrak{n}^{*}$, the annihilator of $z$, which in turn can be canonically identified to $(\mathfrak{n} / z)^{*}$. The natural bilinear form $b(f)$ on $\mathfrak{n} / \mathfrak{z}$ is then transported via $j(f)$ to $z^{\perp} \approx(n / z)^{*}$ giving rise to a bilinear form on the tangent space to the orbit $O(h)$ at the point $f$. This defines a nondegenerate two-form $\omega$ on the orbit $O(h)$, and if $O(h)$ has dimension $2 n, \omega^{n}$ is a volume form on $O(h)$. Finally $\omega^{n}$ defines a measure on $O(h)$ which we view as a measure $\mu_{h}$ on $n^{*}$ concentrated on the orbit $O(h)$. The distribution character $\Theta_{\pi(h)}$ of the representation is then given by

$$
\Theta_{\pi(h)}(\phi)=c^{-1} \int_{O(h)} \hat{\phi}_{1}(y) d \mu_{h}(y)
$$

where $\phi_{1}(x)=\phi(\exp (x))$ and where $\hat{\phi}_{1}$ is the Fourier transform of $\phi_{1}$ and where $c=n ! 2^{n}$ where $2 n=\operatorname{dim} O(h)[11]$.

Let us examine more closely the construction of the two-form $\omega$ on $O(h)$. From the definition of the action of $N$ on $O(h)$ we see at once that the isomorphism of $\mathfrak{n} / \mathfrak{z}$ onto the tangent space to $O(h)$ at a point $f$, that is $z^{\perp} \simeq(\mathfrak{n} / \mathfrak{z})^{*}$, is given by the map $t(b(f))$ of Lemma 3.1 associated to the bilinear form $b(f)$. Therefore the skew two-form $\omega$ at the point $f$ is precisely $b(f)^{*}$ of Lemma 3.1. Now Lemma 3.1 allows us to evaluate the Pfaffian of $b(f)^{*}$, or in other words the ratio of $\omega^{n}$ to the fixed volume $d \nu$ on $z^{\perp} \simeq(\mathfrak{n} / z)^{*}$. The result is that if we normalize 
Lebesgue measure $d \nu$ on $(n / z)^{*} \simeq z^{\perp}$ so that the Fourier transform is isometric on $n / z$ to $(\mathfrak{n} / \mathfrak{z})^{*}$ where we use our preselected choice of Lebesgue measure on $n / z$, and if we translate $\nu$ from $z^{\perp}$ onto $O(h)=H(y)=z^{\perp}+f$, calling this measure $\nu_{h}$, then $d \mu_{h}=|P(h)|^{-1} d \nu_{h}$. Therefore

$$
\Theta_{\pi(h)}(\phi)=c^{-1}|P(h)|^{-1} \int \hat{\phi}_{1}(y) d v_{h}(y)
$$

and so

$$
\phi(e)=\phi_{1}(0)=\int_{3^{*}-v} c^{-1}|P(h)|^{-1}\left(\int \hat{\phi}_{1}(y) d v_{h}(y)\right) d \mu(h)
$$

where $\mu$ is Plancherel measure. But on the other hand,

$$
\int_{b^{*}-\nu}\left(\int \hat{\phi}_{1}(y) d \nu_{h}(y)\right) d z^{*}(h)=\int_{n^{*}} \hat{\phi}_{1}(f) d n^{*}(f)=\phi_{1}(0)=\phi(e)
$$

by the Fourier inversion formula (Plancherel's theorem for the vector group $\mathfrak{n}$ ) and by our choice of normalizations. By comparing these two formulas, we find that Plancherel measure is given by $d \mu(h)=c|P(h)| d z^{*}(h)$ on $z^{*}-\gamma$, and this completes the proof.

5. We discuss a short example which shows that formal degrees of the square integrable representations of a group with compact center need not be bounded away from zero. Indeed according to Example 2 in $\$ 3$, we may find an $\mathfrak{n}$ with $z$ of dimension 2 with a basis $z, w$, and dual basis $z^{*}, w^{*}$ so that the Pfaffian polynomial $P$ is given by $P\left(h_{a, b}\right)=a+\lambda b$ where $h_{a, b}\left(z^{*}\right)=a, h_{a, b}\left(w^{*}\right)=b$ and where $\lambda$ is irrational. Indeed $\mathfrak{n}$ could be taken to be four dimensional with $(x, y, z, w)$ as a basis and $[x, y]=z+\lambda w$ and all other brackets zero. We let $K$ be the group $\left\{\exp \left(n z^{*}+m w^{*}\right): n, m\right.$ integers $\}$ and consider $G=N / K$ which has a compact center. The square integrable representations of $G$ are precisely those of $N$ which are trivial on $K$, that is those $\pi\left(h_{a, b}\right)$ with $a$ and $b$ integral and not both zero. The formal degrees of these representations are the same when viewed as representations of $N$ or as representations of $N / K$ and are given by $d\left(\pi\left(h_{n, m}\right)\right)$ $=|n+\lambda m|$ which is not bounded from zero as $(n, m)$ varies over nonzero lattice points.

6. We turn now to the following situation. Suppose $N$ is nilpotent with center $Z$, and let $\Gamma$ be a discrete uniform subgroup of $N$, and let $U=\operatorname{ind}(\Gamma, N, 1)$ be the natural representation of $N$ by translations on $L_{2}(G / \Gamma)$. Then $U$ decomposes as a discrete direct sum $\sum m_{\pi} \pi$ of irreducible representations of $N$, with finite multiplicities $m_{\pi}$, see [1]. Our object is to determine which square integrable representations $\pi$ occur and with what multiplicity. We shall obtain a very simple formula but in order to state it we need to normalize a volume form on $\mathfrak{n} / \mathfrak{z}$ up to a sign, or equivalently a Haar measure on $N / Z$. We do this by simply declaring that the quotient $N / Z \cdot \Gamma$ has volume equal to one. This determines a Pfaffian 
polynomial $P$ (up to sign) on $z^{*}$. Moreover $\Gamma \cap Z$ is a lattice in $Z$ and $\log (\Gamma \cap Z)$ is a lattice $L$ in $z$; we let $L^{*}$ be the dual lattice in $z^{*}$. The condition for a general representation $\pi$ to occur in $U$ is a kind of complicated integrality condition ([9], [5], [14]); however, for a square integrable representation $\pi(h)$ $=\phi(h), h \in z^{*}-\mathcal{V}$, as given by Theorem 2 , the condition becomes much simpler, and moreover its multiplicity $m_{\pi(h)}$ is given, as one hopes for, by the formal degree as normalized by the choice of Haar measure.

Theorem 7. The square integrable representation $\pi(h), h \in z^{*}-V$, occurs in $U$ if and only if $h \in L^{*}$; moreover, its multiplicity $m_{\pi(h)}$ is $|P(h)|$.

Proof. As usual we proceed by induction on the dimension of $N$, the theorem being obvious in the abelian case. Recall that the existence of the discrete uniform subgroup $\Gamma$ implies that $n$ has the structure of a Lie algebra over the rational number field with a distinguished rational form. We may speak of rational subalgebras $\mathfrak{h} \subset \mathfrak{n}$ and the corresponding rational subgroups $H$ $=\exp (\mathfrak{h})$. Note that a subgroup $H$ is rational if and only if $\Gamma \cap H$ is cocompact in $H$. We first claim that the proof of our theorem may be reduced to the case when the center $Z$ of $N$ has dimension one, for suppose this dimension is larger than one and that $\pi=\pi(h)$ is some square integrable representation where $h \in z^{*}$. It is clear that $h$ must be in $L^{*}$ if $\pi$ is to occur in the representation $U$ since $U$ is trivial on the lattice $\exp (L) \subset Z$. It follows then that if $\pi(h)$ occurs in $U$ or if $h \in L^{*}$, then there is a rational subgroup $Z^{\circ} \neq(e)$ of the center $Z$ on which $\pi$ is trivial. We replace $N$ by $N / Z^{\circ}$ and $\Gamma$ by $\Gamma \cdot Z^{\circ} / Z^{\circ}$ and it is evident that $\pi(h)$ occurs in $L^{2}(N / \Gamma)$ if and only if it occurs in $L^{2}\left(\left(N / Z^{\circ}\right) /\left(\Gamma \cdot Z^{\circ} / Z^{\circ}\right)\right)$ and with the same multiplicity. Finally the Pfaffian polynomial for $N / Z^{\circ}$ which is defined on $\left(z / z^{\circ}\right)^{*} \subset z^{*}$ is the restriction of the corresponding Pfaffian polynomial on $z^{*}$. Thus our assertion for $N$ would follow immediately from the result for $N / Z^{\circ}$ which is of smaller dimension.

Henceforth we assume that $Z$ has dimension one; we fix a nonzero element $x$ not in $z$ but so that $[n, x] \subset z$. We note that it is possible to choose $x$ to be rational [9]. Not only that, but let $z \in z$ be chosen so that $\exp (z)$ is a generator for $\Gamma \cap Z$, and then choose $x$ so that $\exp (z)$ and $\exp (x)$ are generators for $\Gamma \cap Z_{0}$ where $Z_{0}=\exp \left(z_{0}\right), z_{0}=z+(x)$. As usual we form the centralizer $n_{0}$ of $x$ which is of course also a rational ideal of $n$, and we choose our element $y$ not in $n_{0}$ to be rational also. In fact it is wise to choose $y$ somewhat more carefully as follows: the group $\Gamma \cdot N_{0} / N_{0} \subset N / N_{0}$ is a group isomorphic to the integers, and let $\gamma \in \Gamma$ be an element in $\Gamma$ whose image in $\Gamma \cdot N_{0} / N_{0}$ is a generator. Then let $y$ be $\log (\gamma)$, and then $[x, y] \in z$ and indeed $[x, y]=r z$ where $r$ is a rational number. Indeed since $x, y$, and $z$ span a three dimensional rational Heisenberg algebra $\mathfrak{h}$, and since $\Gamma \cap H$ is a discrete subgroup generated by $\exp (x), \exp (y)$ and $\exp (z)$, one can conclude from the known structure of such groups [1] that the rational number $r$ is an integer. 
The square integrable representations of $N$ are parameterized by $0 \neq h \in z^{*}$ or equivalently by $h(z)=s$ which is a nonzero real number. Let $\pi(s)$ be the corresponding representation. As we have already noted $s$ must be an integer if $\pi(s)$ is to occur in $U$, and the $\pi(n), n \in \mathbf{Z}$, are the representations corresponding to points in the lattice $L^{*} \subset z^{*}$. Let $P$ be the Pfaffian polynomial on $z^{*}$ normalized as in the discussion preceding the theorem; then $P$ can be viewed as a function of $s$, and indeed is $c_{k} s^{k}$ for some constant $c_{k}$ where $2 k+1$ is the dimension of $n$. Thus we must show that $\pi(s)$ occurs in $U$ if and only if $s=n$ is integral and that $\pi(n)$ occurs with multiplicity $\left|c_{k} n^{k}\right|$ (and hence in particular that $c_{k}$ is an integer).

Let us consider the group $N_{0}$ and the discrete subgroup $\Gamma_{0}=\Gamma \cap N_{0}$ and the corresponding representation $U_{0}$. We know from our earlier discussion that $z_{0}=z+(x)$ is the full center of $n_{0}$, and $\{z, x\}$ is a basis for $z_{0}$ such that $\exp (z)$ and $\exp (x)$ are a basis for $\Gamma \cap Z_{0}$. We may parameterize $f_{0} \in z_{0}^{*}$ by its values $f_{0}(z)=s$ and $f_{0}(x)=t$. The Pfaffian polynomial $P_{0}$ for $N_{0}$ is a function on $z_{0}^{*}$ and hence is really a polynomial in $s$ and $t$. We have already seen that $P_{0}$ does not depend on $t$ (since $n_{0}$ admits an automorphism fixing $z$ and moving $x$ to $x+a z$ for any $a)$. Thus $P_{0}(s, t)=c_{k-1} s^{k-1}, k-1$ being the proper degree of homogeneity. The normalization is determined by a choice of volume form $\omega_{0}$ on $n_{0} / z_{0}$ so that $N_{0} / Z_{0} \cdot \Gamma_{0}$ has volume one. From the way $x$ and $y$ were chosen relative to $\Gamma$ it is evident that the proper normalization of a volume form $\omega$ on $\mathfrak{n} / \mathfrak{z}$ so that $N / \Gamma \cdot Z$ has volume one is achieved by setting $\omega\left(x, y, u_{1}, \ldots, u_{2 k-2}\right)=$ $\omega_{0}\left(u_{1}, \ldots, u_{2 k-2}\right)$ with $u_{i} \in \mathfrak{n}_{0} / z_{0}$. Now since $[x, y]=r z$, a simple computation shows that $P$ and $P_{0}$ are related by $P(s)=P_{0}(s, t) r s$ so that $P(s)=r c_{k-1} s^{k}$ and hence $c_{k}=r c_{k-1}$.

Now by induction our theorem is true for $N_{0}$; the square integrable representations of $N_{0}$ are parameterized by $z_{0}^{*}-V_{0}$ where $V_{0}$ is the zero set of $P_{0}$, or in other words by pairs $(s, t)$ of real numbers with $s \neq 0$. The lattice $L_{0}^{*} \subset z_{0}^{*}$ consists of those $(s, t)$ which are integral by our choices of $z$ and $x$, and the square integrable representation $\pi_{0}(s, t)$ occurs in $U_{0}$ if and only if $s$ and $t$ are integers, and it occurs with multiplicity $m_{0}(s, t)=\left|c_{k-1} s^{k-1}\right|$. Hence in particular $c_{k-1}$ is an integer and hence so is $c_{k}=r c_{k-1}$.

If $m(s)$ is the multiplicity with which $\pi(s)$ occurs in $U$ the argument in $[9, \mathrm{p}$. 153] gives an algorithm for determining $m(s)$ in terms of the $m_{0}(s, t)$. More precisely, since $\pi_{0}(s, t)$ induces $\pi(s)$ we have to look at the entire $N$-orbit of some $\pi_{0}(s, t)$; but this consists of the $\pi_{0}(s, t)$ as $t$ varies over $R$, with $s$ fixed. We let $A^{\prime}(s)$ be the set of all such representations which occur in $U_{0}$ and by induction this consists of the $\pi_{0}(s, t)$ with $t$ integral (if $s$ is integral) and the void set if $s$ is not integral. We note then that $\Gamma$ operates on $A^{\prime}(s)$ and we let $A(s)$ be a set of representatives. The formula is then

$$
m(s)=\sum m_{0}(s, t) \quad(t \in A(s)) .
$$


This already tells us that $m(s)>0$ if and only if $s$ is integral which is the first part of the theorem telling us which representations $\pi(s)$ occur in $U$. Moreover, we know precisely how $\Gamma$ operates on this set, for $\Gamma_{0}=N_{0} \cap \Gamma$ operates trivially and so we only have to look at $\Gamma / \Gamma_{0}$ which has for generator the image of $\exp (y)$. Since $[x, y]=r z$, it follows immediately that the action of this generator is $\pi_{0}(s, t) \rightarrow \pi_{0}(s, t+s r)$. Therefore, we may pick $A(s)=\{(s, t)$ with $t \in \mathbf{Z}, 0 \leq t$ $<s r\}$ which has $s r$ elements. Since $m_{0}(s, t)=\left|c_{k-1} s^{k-1}\right|$ (independent of $t$ ), the formula (*) immediately yields $m(s)=\left|(r s) c_{k-1} s^{k-1}\right|$ which by inspection is the absolute value of the Pfaffian polynomial $P(s)$. This concludes the inductive step and the proof of Theorem 7.

\section{REFERENCES}

1. L. Auslander et al., Flows on homogeneous spaces, Ann. of Math. Studies, no. 53, Princeton Univ. Press, Princeton, N.J., 1963. MR 29 \#4841.

2. J. Dixmier, Sur les représentations unitaires des groupes de Lie nilpotents. II, Bull. Soc. Math. France 85 (1957), 325-388. MR 20 \# 1928.

3. J. M. G. Fell, The dual space of $C^{*}$-algebras, Trans. Amer. Math. Soc. 94 (1960), 365-403. MR 26 \# 4201.

4. Harish Chandra, Representations of semi-simple Lie groups. II, Trans. Amer. Math. Soc. 76 (1954), 26-65. MR 15, 398.

5. R. Howe, On Frobenius reciprocity for unipotent groups over Q, Amer. J. Math. 93 (1971), 163-172.

6. A. A. Kirillov, Unitary representations of nilpotent Lie groups, Uspehi Mat. Nauk 17 (1962), no. 4 (106), 57-110 = Russian Math. Surveys 17 (1962), no. 4, 53-104. MR 25 \#5396.

7. A. Kleppner and R. Lipsman, The Plancherel formula for group extensions (to appear).

8. G. WV. Mackey, Induced representations of locally compact groups. I, Ann. of Math. (2) 55 (1952), 101-139. MR 13, 434.

9. C. C. Moore, Decomposition of unitary representations defined by discrete subgroups of nilpotent groups, Ann. of Math. (2) 82 (1965), 146-182. MR 31 \#5928.

10. The Plancherel formula for non-unimodular groups, Abstract for the Internat. Congress on Functional Analysis, University of Maryland, College Park, Md., 1971.

11. L. Pukanszky, On characters and the Plancherel formula of nilpotent groups, J. Functional Analysis 1 (1967), 255-280. MR 37 \#4236.

12.— Leçons sur les représentations des groupes, Monographie de la Société Mathématique de France, no. 2, Dunod, Paris, 1967. MR 36 \#311.

13.— Unitary representations of solvable Lie groups, Ann. Sci. Ecole Norm. Sup. 4 (1971), $464-608$.

14. L. Richardson, Decomposition of the $L_{2}$ space of a general compact nilmanifold, Amer. J. Math. 93 (1971), 173-190. MR 44 \# 1771.

15. M. A. Rieffel, Square integrable representations of Hilbert algebras, J. Functional Analysis 3 (1969), 265-300. MR 39 \#6094.

16. I. Satake, Unitary representations of a semi-direct product of Lie groups on d-cohomology spaces, Math. Ann. 190 (1971), 177-202.

17. N. Tatsuuma, The Plancherel formula for non-unimodular locally compact groups, J. Math. Kyoto Univ. 12 (1972), 179-261.

18. A. A. Kirillov, Plancherel's measure for nilpotent Lie groups, Funkcional. Anal. i Priložen. 1 (1967), no. 4, 84-85. (Russian) MR 37 \#347.

Department of Mathematics, University of California, Berkeley, California 94720 